\title{
DVB-T2: New Signal Processing Algorithms for a Challenging Digital Video Broadcasting Standard
}

\author{
Mikel Mendicute, Iker Sobrón, Lorena Martínez and Pello Ochandiano \\ Signal Theory and Communications Area \\ Mondragon Goi Eskola Politeknikoa \\ University of Mondragon, \\ Spain
}

\section{Introduction}

Digital Video Broadcasting-Terrestrial (DVB-T) is the most widely deployed digital terrestrial television system worldwide with services on air in over thirty countries. In order to increase its spectral efficiency and to enable new services the DVB consortium has developed a new standard named DVB-T2. A nearly definitive specification has already been published as a BlueBook as well as an implementation guideline, where the structure and main technical novelties of the standard have been defined. The imminent publication of the final DVB-T2 standard will give rise to the deployment of new networks and commercial products.

The differences between the original DVB-T and the new DVB-T2 standards are many and important. The latest coding, interleaving and modulation techniques have been included in this large and flexible specification to provide capacity and robustness in the terrestrial transmission environment to fixed, portable and mobile terminals. Multiple-input multipleoutput (MIMO) techniques, low-density parity-check codes (LDPC), rotated constellations, new pilot patterns or large interleaving schemes are the most remarkable signal processing algorithms that have been included to overcome the limitations of the much simpler DVB-T broadcasting standard.

This chapter focuses on the mentioned new algorithms and the opportunities that arise from a signal processing perspective. New transmission and reception techniques are proposed which can be used to enhance the performance of DVB-T2, such as iterative demapping and decoding, new antenna diversity schemes or more efficient channel estimation algorithms. Furthermore, the performance of the new standard is analyzed and evaluated through simulations focusing on the aforementioned algorithms. The behaviour of the standard is specially studied in single-frequency networks (SFN), where the vulnerability of the former standard is prohibitive when destructive interferences arise.

The chapter first describes the main architecture and limitations of the original DVB-T specification. The physical layer of the new DVB-T2 standard is then defined, emphasizing the main differences in comparison to its predecessor. The next section of the chapter proposes and analyzes iterative demapping and decoding techniques at reception which can profit from the benefits of the new LDPC codes. Multi-antenna transmission and reception is

Source: Digital Video, Book edited by: Floriano De Rango,

ISBN 978-953-7619-70-1, pp. 500, February 2010, INTECH, Croatia, downloaded from SCIYO.COM 
next studied, evaluating the benefits of the antenna diversity schemes proposed by the standard on the performance of the system. Channel estimation issues are analyzed in the following section, presenting a bidimensional estimation algorithm which is specially interesting due to the mobility requirements of the new standard and to the plethora of pilot patterns that have been defined. Last, two relevant issues of the new standard are analyzed and evaluated through simulations: the rotated constellation-based transmission and the performance in SFN scenarios. Provided results show the behavior of the new DVB-T2 standard and the improvement achievable by applying the new signal processing algorithms proposed throughout this chapter.

\section{DVB-T and its limitations}

The DVB-T terrestrial digital video broadcasting standard (ETSI, 1997) is replacing the former analogue systems in many countries around the world. The benefits of digital coding and transmission techniques allow perfect signal recovery in all the serviced areas avoiding the effects of the wireless channel and noise. Considering the physical level of the communications, the digital data sequences, which contain MPEG video, audio and other information streams, are transmitted using coded orthogonal frequency division multiplexing (COFDM) modulation. The information bits are coded, interleaved, mapped to a quadrature amplitude modulation (QAM) constellation and grouped into blocks. All the symbols in a block are transmitted simultaneously at different frequency subcarriers using an inverse fast Fourier transform (IFFT) operation. The number of IFFT points, which can be either $2048(2 \mathrm{~K})$ or $8192(8 \mathrm{~K})$, determines the transmission mode and the number of the available subcarriers in the transmission bandwidth. Some of these subcarriers are not used to allow for guard frequency bands whereas others are reserved for pilot symbols, which are necessary to acquire the channel information required for signal recovery.

Fig. 1 shows the main diagram of a DVB-T transmitter. As it can be seen, the data bit stream is scrambled, processed by an outer Reed-Solomon (RS) coder, an interleaver and an inner convolutional coder. The first coding stage removes possible error floors at high signal-tonoise (SNR) values, whereas the second reduces the bit error rate (BER) at the receiver by including more redundant information depending on the selected coding rate (CR), which can range from $1 / 2$ to $5 / 6$. The coded information bits are interleaved again in order to allocate consecutive bits to different subcarriers. The resulting information bits are then arranged by blocks, mapped and modulated using OFDM, which involves an IFFT operation and the addition of a cyclic prefix to enable a guard interval (GI) that avoids interference between consecutive blocks. The use of coding and interleaving processes over OFDM provides an efficient and robust transmission method in multipath scenarios enabling time and frequency diversity.

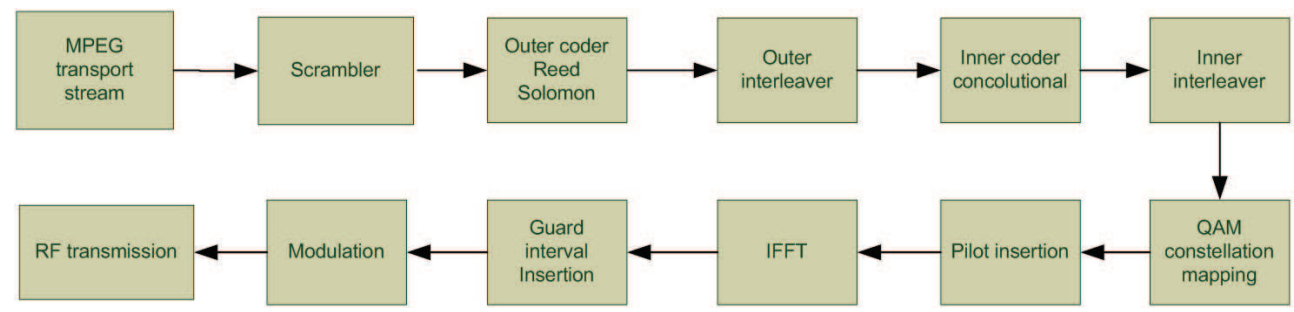

Fig 1. Elementary transmission chain of DVB-T. 
Despite the many benefits achieved by the deployment of the DVB-T network, its limitations became clear from the beginning. First, the number and bit rates of the transmitted channels are limited in comparison with new wireless transmission techniques. A new standard was soon required to broadcast more channels and high-definition television (HDTV) using the same frequency spectrum. Second, a new information system was required to allow more interaction with the user. Third, the DVB-T standard, which had been designed for fixed scenarios, had a very bad performance in mobile or portable environments, so it could not be properly implemented in scenarios such as moving vehicles. Last but not least, the deployment of the DVB-T network has been and still is a true nightmare in SFN scenarios, where interferences between repeaters, which transmit the same information on the same frequency bands, may destroy the received signal avoiding its reception in areas with good reception levels.

Considering the new advances in signal processing, modulation and coding, the DVB consortium has published a draft standard named DVB-T2 aiming to extend the capabilities of the aforementioned DVB-T standard.

\section{The new DVB-T2 standard}

Based on recent research results and a set of commercial requirements, the DVB consortium concluded that there were suitable technologies which could provide increased capacity and robustness in the terrestrial environment, mainly for HDTV transmission. Therefore, a new standard named DVB-T2 has been designed primarily for fixed receptors, although it must allow for some mobility, with the same spectrum characteristics as DVB-T. Fig. 2 shows the main stages of a DVB-T2 transmitter, where dashed lines represent optional stages.

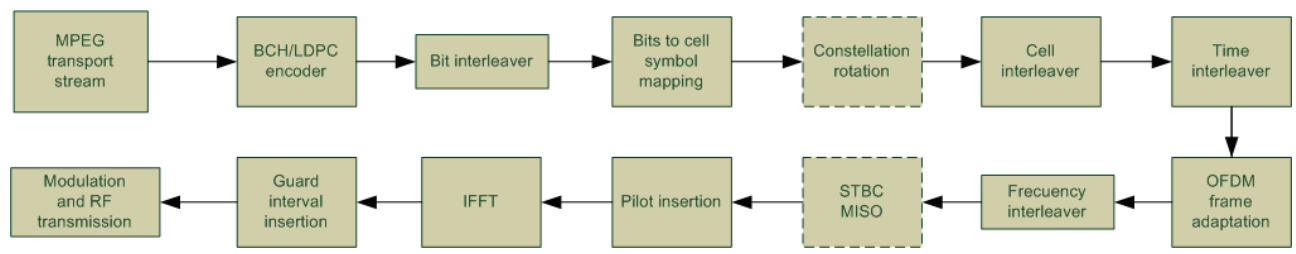

Fig. 2. Elementary transmission chain of DVB-T2.

The first remarkable novelty lies on the error correction strategy, since DVB-T2 uses the same channel codes that were designed for DVB-S2. The coding algorithms, based on the combination of LDPC and Bose-Chaudhuri-Hocquenghem (BCH) codes, offer excellent performance resulting in a very robust signal reception. LDPC-based forward error correction (FEC) techniques can offer a significant improvement compared with the convolutional error correcting scheme used in DVB-T.

Regarding the modulation, DVB-T2 uses the same OFDM technique as DVB-T. Maintaining the $2 \mathrm{~K}$ and $8 \mathrm{~K}$ modes, the new standard has introduced longer symbols with $16 \mathrm{~K}$ and $32 \mathrm{~K}$ carriers in order to increase the length of the guard interval without decreasing the spectral efficiency of the system. The new specification offers a large set of modulation parameters by combining different numbers of carriers and guard interval lengths, making it a very flexible standard as it is shown in Table 1. Furthermore, the highest constellation size has been increased to 256 symbols (256QAM).

As it will be extended in Section 6, another interesting innovation is the introduction of 8 different scattered pilot patterns, whose election depends on the parameters of the current 
transmission. Thus, thanks to all the configurable parameters of the new standard, the modulation can be adapted to the characteristics of the actual transmission, making the most of the spectral efficiency. As it can be seen in Fig. 2, an important innovative feature proposed by the DVB-T2 specification is the use of three cascaded forms of interleaving, which are the following: bit interleaver, time interleaver and frequency interleaver. The aim of all these interleaving stages is to avoid error bursts, giving rise to a random pattern of errors within each LDPC FEC frame.

\begin{tabular}{|c|c|c|}
\hline & DVB-T & DVB-T2 \\
\hline \multirow[t]{2}{*}{ FEC } & $\begin{array}{l}\text { Convolutional + Reed- } \\
\text { Solomon }\end{array}$ & $\mathrm{LDPC}+\mathrm{BCH}$ \\
\hline & $1 / 2,2 / 3,3 / 4,5 / 6,7 / 8$ & $1 / 2,3 / 5,2 / 3,3 / 4,4 / 5,5 / 6$ \\
\hline Modes & QPSK, 16QAM, 64QAM & QPSK, 16QAM, 64QAM, 256QAM \\
\hline Guard intervals & $1 / 4,1 / 8,1 / 16,1 / 32$ & $\begin{array}{c}\text { 1/4, 19/256, } 1 / 8,19 / 128,1 / 16,1 / 32 \\
1 / 128\end{array}$ \\
\hline FFT size & $2 \mathrm{~K}, 8 \mathrm{~K}$ & $1 \mathrm{~K}, 2 \mathrm{~K}, 4 \mathrm{~K}, 8 \mathrm{~K}, 16 \mathrm{~K}, 32 \mathrm{~K}$ \\
\hline Scattered pilots & $8 \%$ of total & $1 \%, 2 \%, 4 \%$ and $8 \%$ of total \\
\hline Continual pilots & $2.6 \%$ of total & $0.35 \%$ of total \\
\hline
\end{tabular}

Table 1. Available modes in DVB-T and DVB-T2.

On the other hand, a new technique called rotated constellations and Q-delay (RQD) is provided as an option, which comes to offer additional robustness and diversity in challenging terrestrial broadcasting scenarios. Furthermore, a mechanism has been introduced to separately adjust the robustness of each delivered service within a channel in order to meet the required reception conditions (in-door antenna/roof-top antenna, etc.). DVB-T2 also specifies a transmitter diversity method, known as Alamouti coding, which improves coverage in small scale single-frequency networks.

Finally, the DVB-T2 standard takes into account one of the main drawbacks of OFDM, the peak to average power ratio (PAPR) of the signal and its effects on the transmitter equipments. High power peaks are usually generated by OFDM transmission leading to distortions at the amplifiers, thus minimizing their efficiency. Two techniques have been included in the standard to limit the PAPR without degrading the transmitted signal: carrier reservation and active constellation extension. The first reserves some subcarriers that can be used to correct the PAPR level of the transmitted signal whereas the latter achieves the same effects modifying the QAM constellation without degrading the signal recovery at reception.

Fig. 3 shows the comparative performance of DVB-T and DVB-T2 for similar communication parameters. The BER at the output of the inner decoder has been considered in all the simulation results provided in this chapter. In order to allow a fair comparison of both standards, a quasi error free $(\mathrm{QEF})$ of $\mathrm{BER}=2 \cdot 10^{-4}$ and $\mathrm{BER}=10^{-7}$ must be considered for DVB-T and DVB-T2 after convolutional and LDPC decoders, respectively. If these QEF reference values are analyzed, a gain of $6 \mathrm{~dB}$ can be established between the two standards in an additive white Gaussian noise (AWGN) channel model and nearly $4 \mathrm{~dB}$ in a Rayleigh channel. The code rates have been selected in order to approach equivalent systems. 

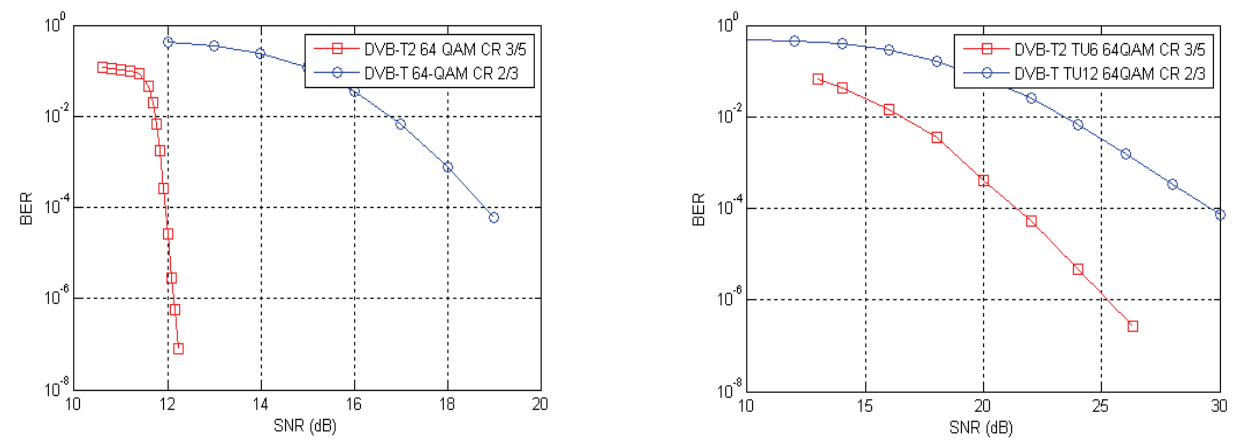

Fig. 3. BER performance of DVB-T and DVB-T2 systems in AWGN (a) and Rayleigh (b) channels.

All the DVB-T2 simulation results presented in this chapter have been obtained using the following transmission parameters: FEC frame length of 16200 symbols; 2K OFDM mode and a guard interval of $1 / 4$.

\section{Iterative demapping and decoding of LDPC codes}

As it has been stated, one of the major innovations of DVB-T2 lies on the selected channel coding techniques. The coding schemes used in first-generation digital television standards (Reed Solomon and a convolutional code for outer and inner coding, respectively) have been replaced by $\mathrm{LDPC}$ and $\mathrm{BCH}$ codes in the second generation of the digital television standards published to date, such as DVB-S2 and DVB-T2. The main advantage of LDPC codes is that they provide a performance which approaches the channel capacity for many different scenarios, as well as the linear algorithms that can be used for decoding. Actually, the efficiency improvement provided by DVB-T2 in comparison with DVB-T is mainly based on these new coding and interleaving schemes.

\subsection{Basics of BICM schemes and SISO processing}

LDPC codes are commonly decoded by a soft-input soft-output (SISO) algorithm which iteratively computes the distributions of variables in graph-based models. It has been published under different names and models, such as the sum-product algorithm (SPA), the belief propagation algorithm (BPA) or the message-passing algorithm (MPA). The decoding of the information bits is based on the computation of the a posteriori probability (APP) of a given bit in the transmitted codeword $c=\left[c_{0} c_{1} \ldots c_{n-1}\right]$ subject to the received symbol vector $y$ $=\left[\begin{array}{lll}y_{0} & y_{1} \ldots & y_{n-1}\end{array}\right]$. Therefore, the APP ratio must be computed. A numerically more stable version called log-likelihood ratio (LLR) is commonly used as defined in the following equation:

$$
a_{l}=\ln \left[\frac{P\left(c_{l}=1\right)}{P\left(c_{l}=0\right)}\right]
$$

where $P\left(c_{l}=x\right)$ denotes the probability of codeword bit $c_{l}$ having the value $x$. 
DVB-T2 implements a bit-interleaved LDPC coded modulation (BILCM) scheme, which has been employed in many broadcasting and communication systems. BILCM is a special case of a more general architecture named bit-interleaved coded modulation (BICM). It was proposed by Zehavi (Zehavi, 1992) and consists of coding, bit-wise interleaving and constellation mapping. Several studies have shown that BICM presents an excellent performance under fading channels (Li et a., 1998).

The capacity of BICM schemes depends on several design parameters. An information theory point of view is given in (Caire et al., 1998) for input signals constrained by a specific complex constellation $\chi$. Considering the simplest discrete-time memoryless complex AWGN channel modelled as $y=x+n$, where $y, x$ and $n$ denote the output value, the input sample and the complex Gaussian noise sample with zero mean and variance $N_{0} / 2$ for each real and imaginary part respectively, and being $N_{0}$ the noise spectral power density. The channel capacity can be evaluated as follows for an $m$-order modulation in case of a coded modulation $(\mathrm{CM})$ system.

$$
C=m-E_{x, y}\left[\log _{2} \frac{\sum_{z \in \chi} p(y \mid z)}{p(y \mid x)}\right]
$$

However, in case of applying a BICM system, the channel capacity is always lower because each bit level is demapped independently.

$$
C^{\prime}=m-\sum_{i=1}^{m} E_{b, y}\left[\log _{2} \frac{\sum_{z \in \chi} p(y \mid z)}{\sum_{z \in \chi_{i}^{(b)}} p(y \mid z)}\right]
$$

where $\chi_{i}^{(b)}$ denotes the subset of $\chi$ whose corresponding i-th bit value is $b \in\{0,1\}$.

Therefore, BICM is a sub-optimal scheme since $C \geq C^{\prime}$.

\subsection{Iterative demapping and decoding of LDPC codes for DVB-T2 receivers}

This section describes the application of novel iterative demapping and decoding algorithms over BILCM for DVB-T2 receivers. This iterative receiver scheme, named BILCM with iterative demapping (BILCM-ID) was firstly described in (Li et al., 1998) and (Li et al., 2002), where it has been shown that BICM schemes are sub-optimal from a information theoretical point of view. Nevertheless, BICM-ID schemes are optimal because, although the bit-levels are not demapped independently, they are fed back to assist demapping other bits within the same symbol.

The BILCM-ID model is represented by the block diagram of Fig. 4. Soft information given by SISO blocks, such as the LDPC decoder or the soft demapper, is usually fed back from one block to another. In the research described in this paper, the demapping process is fed with soft values from the SISO LDPC decoder, exchanging information iteratively between the two blocks. The soft demapper uses the extrinsic information generated by the LDPC decoder as a priori information for the demapping process.

In general, the complex received signal at symbol index $j, r_{j}$, can be expressed as a $r_{j}=h_{j} s_{j}+$ $n_{j}$. The demapping stage consists of two stages. First, the soft demapper computes $m$ a posteriori probabilities (one for each point of the modulated constellation) for every symbol received from the channel as it is shown in the following equation: 


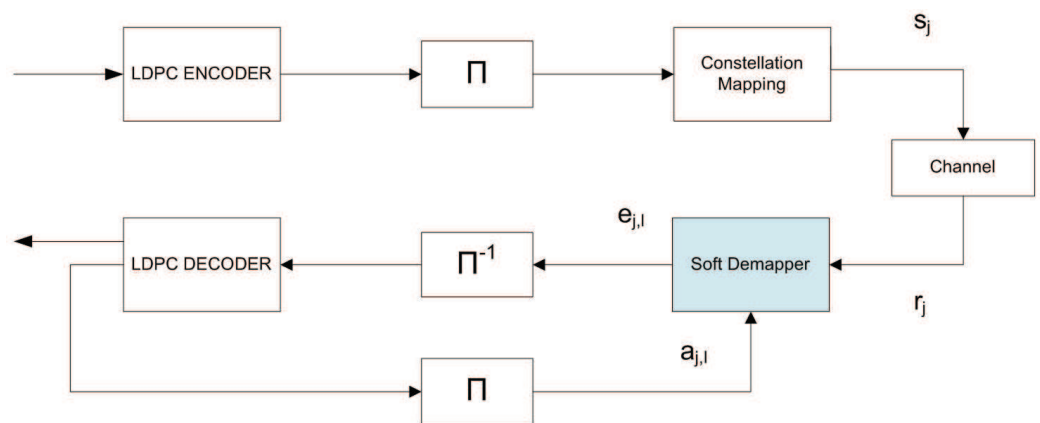

Fig. 4. Transmitter and receiver modules of a BILCM-ID system.

$$
f\left(y \mid s_{j}\right)=\exp \left\{\frac{-\left|r_{j}-h_{j} s_{j}\right|^{2}}{N_{0}}\right\}
$$

In the second step, extrinsic LLRs $e_{j, l}$, where $l=[1, \ldots, \mathrm{m}]$, are calculated as follows:

$$
e_{j, l}=\frac{\sum_{s_{j} \in \chi^{1}} \exp \left\{\frac{-\left|r_{j}-h_{j} s_{j}\right|^{2}}{N_{0}}+\sum_{i=1, i \neq l}^{m} s_{j, i} a_{j, i}\right\}}{\sum_{s_{j} \in \chi^{0}} \exp \left\{\frac{-\left|r_{j}-h_{j} s_{j}\right|^{2}}{N_{0}}+\sum_{i=1, i \neq l}^{m} s_{j, i} a_{j, i}\right\}}
$$

where $\chi^{t}$ denotes the signals $s_{j} \in X$ whose $l^{\text {th }}$ bit has the value $t \in\{0,1\}$. As it is shown in Equation (5), the use of a priori information tries to enhance the reliability of symbol probabilities.

\subsection{Simulation results}

The simulation-based BER performance of iterative demapping in DVB-T2 receivers is analyzed in this section for different modulation orders, code rates and channel models. Bitinterleaving is restricted within one LDPC FEC codeword as defined by the standard. As can be seen in Fig. $5 \mathrm{a}$ and $5 \mathrm{~b}$, iterative processing always provides a gain in comparison to a single demapping and decoding stage. Nevertheless, it can be seen that the performance of BILCM-ID systems has a strong dependence on the code rate and the modulation order. The rationale behind this dependence is that the capacity gap between CM and BICM systems decreases as the coding rate grows, whereas it grows with the constellation order. Furthermore, several studies have proved that Gray mapping makes such gap negligible at high coding rates.

Fig $5 \mathrm{a}$ depicts the simulation results for code rate $1 / 2$ using 16QAM and 64QAM constellations over an AWGN channel. On the other hand, Fig. 5b shows results for different code rates and a 64QAM constellation over a Typical Urban 6 (TU6) channel (COST207, 1989). Blue lines correspond to standard reception without iterative demapping, whereas red lines represent 3 demapping-decoding iterations. For all the simulation results provided 

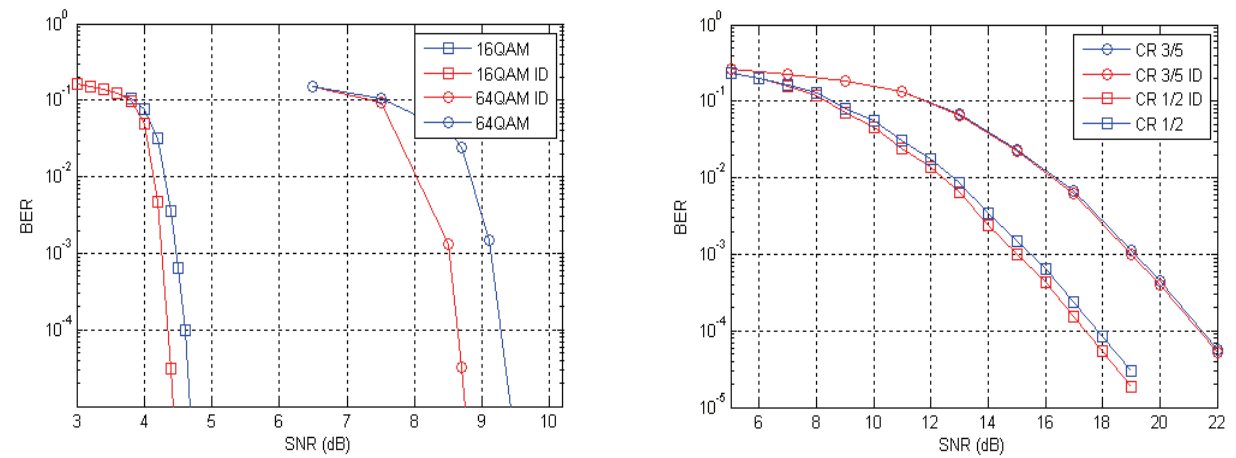

Fig. 5. BER versus SNR performance curves for iterative and non-iterative demapping and decoding in AWGN (a) and TU6 (b) channels.

in this chapter, the LDPC decoder runs a maximum of 50 internal iterations for each detection stage.

Simulation results confirm the values expected from the information theoretical analysis described previously: the gain provided by iterative demapping is insignificant for high coding rates and increases as the constellation order grows. It can be seen in Fig. 5a that there is a gain of $0.25 \mathrm{~dB}$ with 16QAM and $0.6 \mathrm{~dB}$ with 64QAM at a BER of $10^{-4}$.

As has been said, the feedback to the demapper is usually performed when the decoding process has finished after 50 iterations. However, the number of decoder iterations in each demapping stage can be modified in order to offer the best error correcting performance and keep the same maximum number of overall iterations (50 decoder iterations $\times 3$ demapping stages). This new design is called irregular iterative demapping (ID-I) and is specially interesting to design efficient iterative demapping receivers.

Fig. 6a describes the performance of the LDPC decoder for 3 demapping iterations (64QAM over AWGN channel) at a specific SNR value of $8.75 \mathrm{~dB}$, both for the regular case and the irregular one. The implemented irregular demapping approach performs 25, 75 and 50 decoding iterations at the first, second and third demapping stages, respectively. Regarding the regular case, it can be seen that the LDPC decoder converges at the first demapping
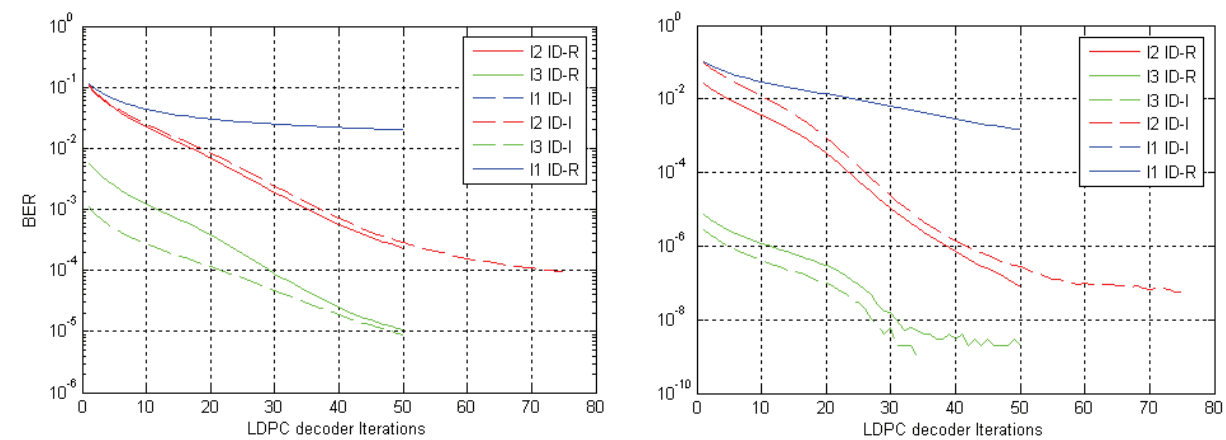

Fig. 6. BER versus LDPC decoder iterations for regular and irregular iterative demapping strategies at SNR value of $8.75 \mathrm{~dB}$ (a) and at SNR value of $9.1 \mathrm{~dB}(\mathrm{~b})$. 
iteration, which means that the BER performance will not improve if more decoder iterations are carried out. Moreover, the BER gain reached at the first demapping iteration is not too high in comparison with the second one which has not converged at the end of the decoding process, which means it could be extended up to a point close to the convergence value.

On the other hand, the irregular approach performs more decoder iterations in the second demapping stage and consequently the error correcting performance can be improved at the end of the decoding process. Fig. $6 \mathrm{~b}$ demonstrates that irregular iterative demapping gain is even larger in higher SNR values. For example, at a BER value of about $10^{-9}$, the third demapping iteration has converged in the $35^{\text {th }}$ decoder iteration for the regular case, whereas the irregular one has not.

\section{MISO transmission and receiver diversity}

MIMO wireless communication systems are based on signal processing with multiple antennas at both transmitter and receiver side. Theoretical works such as (Foschini \& Gans, 1998) and (Telatar, 1999) have shown that the use of multiple antennas can increase the limits of the channel capacity. Wireless telecommunications systems such as WLAN 802.11n or WMAN 802.16e have included MIMO techniques in their newest specitifications. However, the new DVB-T2 standard only proposes the use of several antennas at one side of the transmission. These subsets of MIMO systems are called multiple-input single-output (MISO) and single-input multiple-output (SIMO) schemes. The first, which corresponds to multiple transmit and only one receive antenna, offers transmit diversity, whereas the latter, which includes multiple receive antennas, offers receive diversity.

\subsection{The DVB-T2 MISO transmission scheme}

The DVB-T2 standard describes a transmit diversity method with two antennas based on a modified Alamouti coding scheme (Alamouti, 1998). The coding algorithm is generically called space-frequency block coding (SFBC) since the Alamouti scheme is used in spatial and frequency domain as is depicted in Fig. 7. As can be seen, the Alamouti SFBC approach processes the symbols pairwise, sending the original values $\left(\left[a_{0}, b_{0}\right]\right.$ for the first symbol pair) at one of the antennas and modified values $\left(\left[-b_{0}^{*}, a_{0}^{*}\right]\right)$ at the other one, thus increasing the transmit diversity while keeping the symbol rate.

The received complex values for the first pair of MISO cells are given by:

$$
\begin{aligned}
& R_{1}=H_{1} a_{0}-H_{2} b_{0}{ }^{*}+N_{1} \\
& R_{2}=H_{1} b_{0}+H_{2} a_{0}{ }^{*}+N_{2}
\end{aligned}
$$

where $H_{1}$ and $H_{2}$ denote the channel transfer gains from transmitters 1 and 2 to the receiver, while $N_{1}$ and $N_{2}$ are the noise samples. These equations can be represented in matrix notation as follows:

$$
\left(\begin{array}{c}
R_{1} \\
R_{2}^{*}
\end{array}\right)=\left(\begin{array}{cc}
H_{1} & -H_{2} \\
H_{2}{ }^{*} & H_{1}^{*}
\end{array}\right)\left(\begin{array}{c}
a_{0} \\
b_{0}{ }^{*}
\end{array}\right)+\left(\begin{array}{c}
N_{1} \\
N_{2}{ }^{*}
\end{array}\right)
$$

which makes the decoding process simpler at the receiver. This method aims to improve the coverage and robustness of the reception in SFN networks, so that the transmitters of two 
SFN cells form a "distributed" MISO system, providing space and frequency diversity. Fig. 8 shows the BER performance of the MISO DVB-T2 system in comparison to the SISO system with a TU6 channel model of six paths (COST207, 1989). This channel corresponds to a multipath propagation scenario with Rayleigh fading (Patzold, 2002). The diversity gain for a 64QAM mode with LDPC code rate $3 / 5$ is around $5 \mathrm{~dB}$ just above the QEF value of $10^{-7}$ after LDPC decoder as is specified in the DVB-T2 implementation guidelines (DVB, 2009).

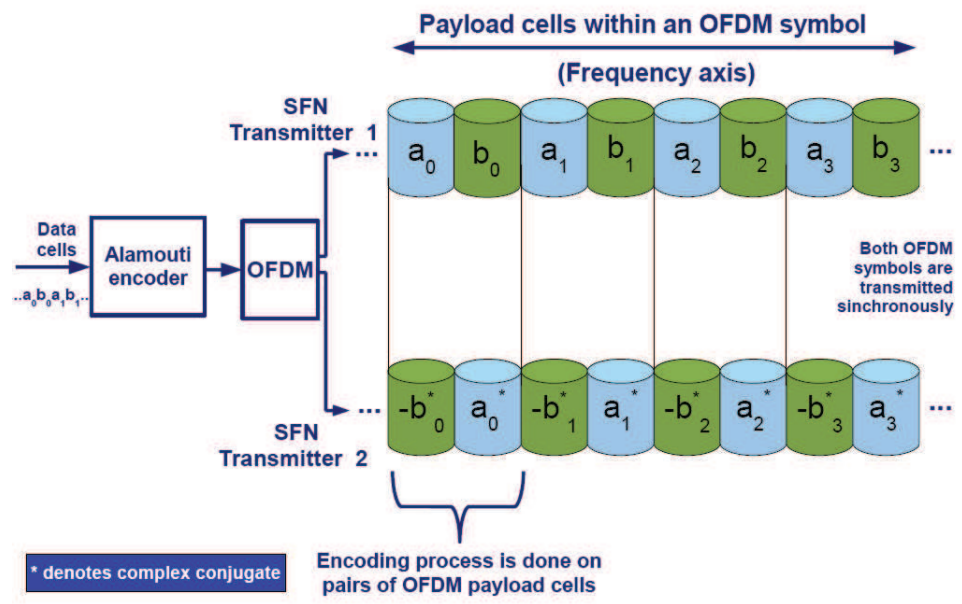

Fig. 7. MISO encoding in DVB-T2 systems.

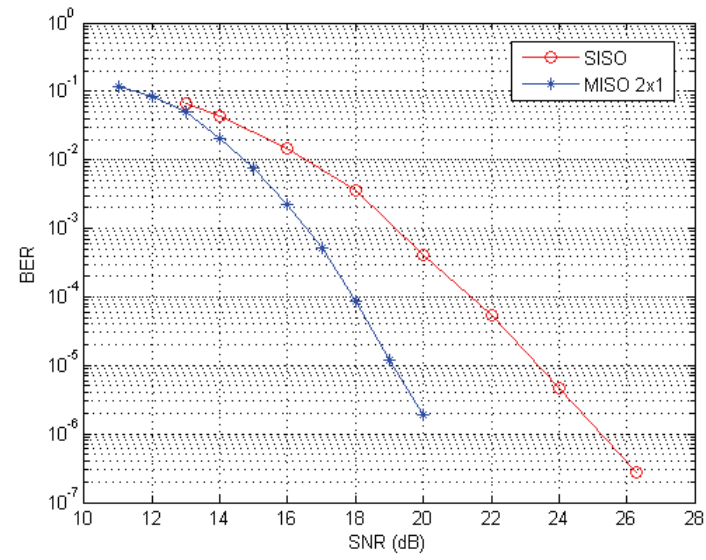

Fig. 8. Performance of MISO transmission for a 64QAM constellation and CR 3/5 over TU6 channel in DVB-T2.

\subsection{Effects of receive diversity}

The DVB-T2 standard only includes requirements for transmission, so the signal processing at the receiver can be freely modified to improve the performance of the overall system. 
There are several receiver techniques that can be applied, such as iterative and non iterative algorithms of equalization and decoding (Proakis, 1995) or receive diversity techniques (Jakes, 1974). This section will focus on a simple but effective receive diversity technique called maximal ratio combining (MRC) which performs a weighted linear combination of the input signals. The signal recovery process can be represented by the following equation:

$$
R_{k}=\sum_{i=1}^{N} H_{k, i}{ }^{*} R_{k, i},
$$

where $R_{k, i}$ and $H_{k, i}^{*}$ are the received signal and the complex conjugate of the channel transfer function between the transmitter and the $i$-th receiver antenna for subcarrier index $k$. This receiver diversity method maximizes the output SNR and has been widely studied for DVB$\mathrm{T}$ in portable and mobile scenarios (Levy, 2004). Since DVB-T2 is targeted at fixed, portable and mobile scenarios, MRC results a suitable technique to improve the reception quality. Furthermore, it can also be combined with the aforementioned MISO transmission scheme specified in DVB-T2, hence forming a $2 \times 2$ setup.

Fig. 9 depicts the performance of such a $2 \times 2$ transmitter and receiver diversity system in a TU6 channel with the former configuration. An improvement of $6 \mathrm{~dB}$ can be observed at the QEF level in comparison to the $2 \times 1$ MISO system, which is due to antenna array and diversity gains at the receiver. Nevertheless, it involves a greater cost than MISO as part of the receiver chain must be replicated, which can be expensive for consumer products. Consequently, the receive diversity may be targeted to specific equipments, such as mobile or portable receivers and problematic fixed locations.

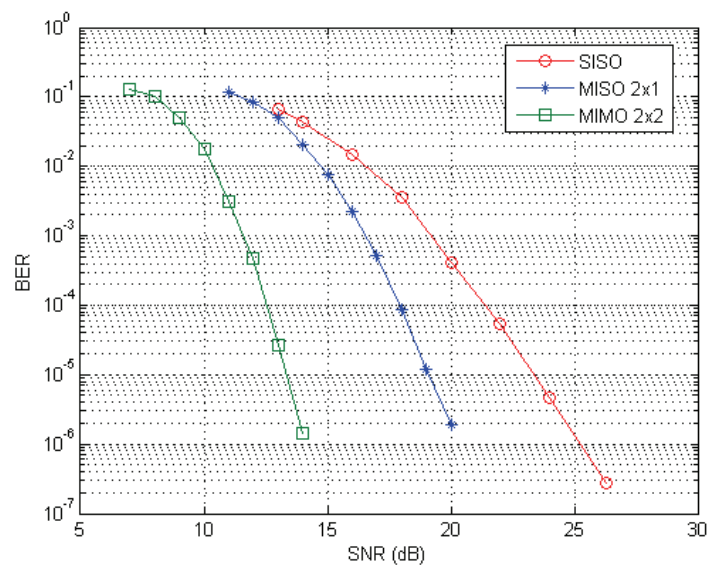

Fig. 9. Performance of the MIMO transmission in DVB-T2.

\section{Channel estimation and tracking}

As has been detailed in previous sections, one of the main innovative aspects of DVB-T2 is the plethora of pilot types and patterns provided, which make channel estimation more flexible. This section describes the pilot structure in more detail and proposes the application of an effective and well-known channel estimation algorithm which can profit from the flexibility and the information provided by all the available pilot subcarriers. 


\subsection{Pilot symbol locations and sets in DVB-T2}

As it has been summarized in Sections 2 and 3, an OFDM frame is composed of a block of symbols whose length depends on the selected transmission mode. For the case of DVB-T, two modes are defined, $2 \mathrm{~K}$ and $8 \mathrm{~K}$, which use 1705 and 6817 of the available subcarriers, respectively. However, only 1512 and 6048 of the subcarriers correspond with information symbols. The rest of subcarriers, named pilots, are commonly used for synchronization and channel estimation. These pilot subcarriers are divided into three groups: continual (which are available at every symbol), scattered (whose location varies for different set of symbols) and transmission parameter signaling (TPS), which are used to acquire information about the format of the signal.

DVB-T2 introduces some remarkable differences in this respect, as it incorporates new reference signals. Several pilot groups have been defined: continual, scattered, edge, P2 and frame-closing pilot cells. The location and amplitudes of the pilots differ with respect to DVB-T and various sets of pilots are applied depending on the transmission parameters. There are eight different scattered pilot patterns (named from PP1 to PP8). Therefore, depending on the FFT size, the length of the guard interval, pilot pattern and the antenna settings, the number of pilot and overall used subcarriers can vary. Furthermore, the continual pilot locations are taken from one or more sets depending on the FFT mode and the pilot positions belonging to each set depend on the scattered pilot pattern in use. Fig. 10 shows the locations of scattered and other pilots for a sample pilot pattern named PP1.

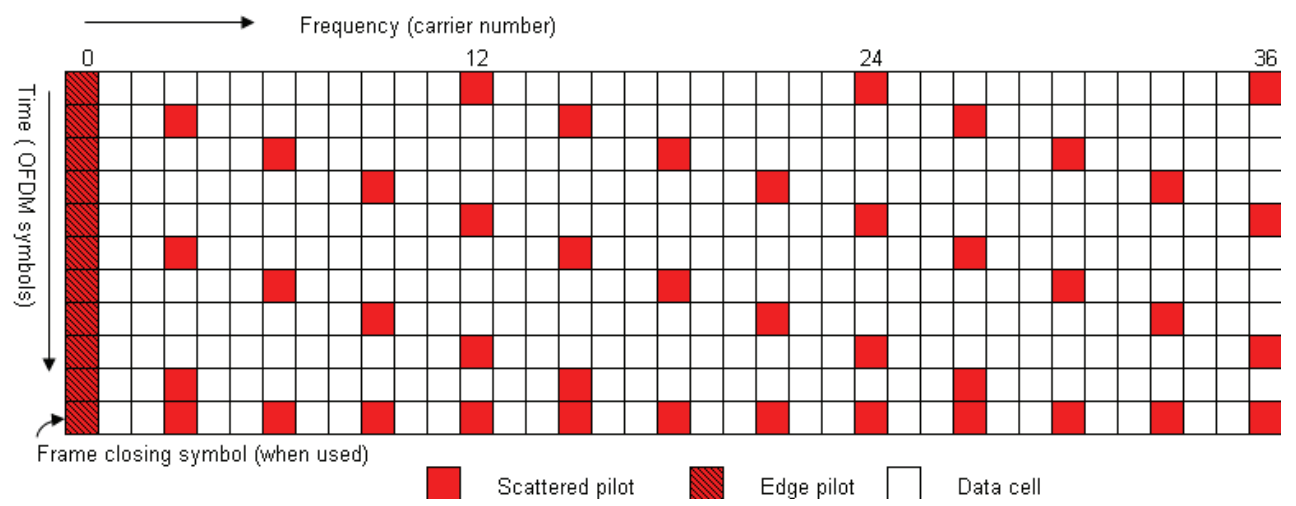

Fig. 10. Sample scattered pilot pattern (PP1) defined by the DVB-T2 standard for SISO transmission.

The edge pilots are inserted in order to allow frequency interpolation up to the edge of the spectrum. P2 pilots are inserted in P2 symbols, which may carry data and are used to transmit signaling information. As can be stated from the previous enumeration, the channel estimator must be a very flexible structure in order to allow for all the possible options and to profit from the information provided by all the pilot symbols. Channel estimation can be divided into two stages: estimation at the pilot subcarriers and interpolation of the intermediate subcarrier gains, both in the frequency (rest of subcarriers) and the time domain (surrounding symbols). Bidimensional Wiener filter-based approaches, such as the one that is described in this section, can perform both stages at once. 


\subsection{Two dimensional channel estimation in DVB-T2}

An efficient and adaptive estimate of the channel can be obtained if the channel estimator processes the information in both time and frequency domain, profiting from the coherence time and bandwidth of the channel, respectively. Such an algorithm based on pilot subcarriers and Wiener filtering is studied in (Necker \& Stüber, 2004) for an OFDM system, showing a good trade-off between low complexity and speed of convergence. The structure of a two-dimensional estimator is too complex for practical implementation but it is possible to replace a 2D-filter by two 1D-filters, one running over the OFDM symbols (time domain) and the other over the subcarriers of an OFDM symbol (frequency domain), to simplify the implementation.

Given the Wiener design criteria outlined in (Hoeher et al., 1997), the filter coefficients are calculated through the cross-correlation of each of the carriers with the pilots and through the autocorrelation of the pilot carriers. This way, filter weights are generated from the statistics of the channel and the position of the pilots. Fig. 11 shows the performance of this channel estimation algorithm in a DVB-T2 scenario. Fig. 11a shows the value of the channel estimate using several different channel estimation and interpolation algorithms.
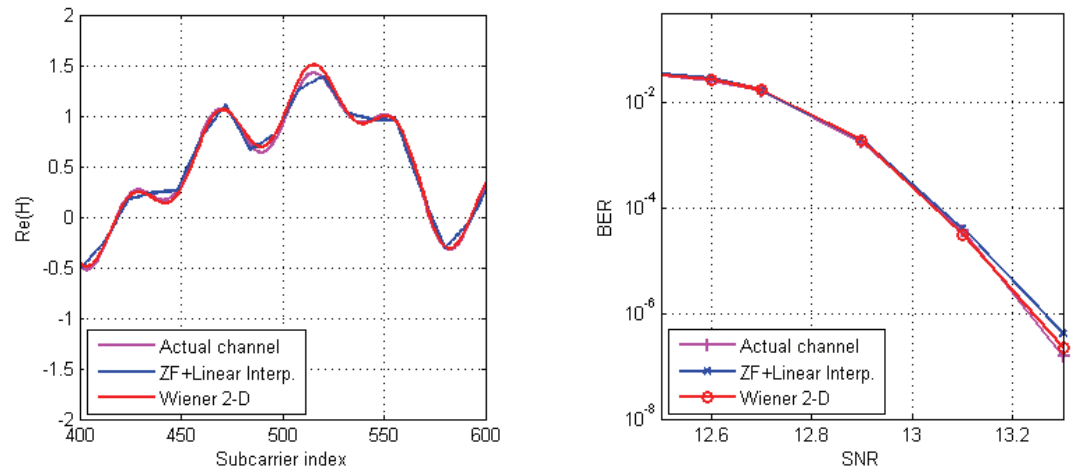

Fig. 11. Simulation results showing the performance of different channel estimation and interpolation schemes: channel value (a) and BER curve (b).

As can be seen in Fig. 11, the Wiener-based bidimensional channel estimator is the one that gets closest to the actual channel, whereas the simplest algorithm, based on zero-forcing and linear interpolation in the frequency-domain, shows to be the worst with a very poor performance. On the other hand, Fig. 11b shows the BER curves of the different estimation approaches for a static TU6 channel. The differences in these results depend on the performance of the channel interpolation method.

\section{Effects of constellation rotation on DVB-T2 reception}

The constellation rotation or RQD operation specified in DVB-T2 aims to increase the diversity order of the DVB-T2 BICM scheme. This technique is comprised of two stages: correlating the in-phase (I) and quadrature (Q) components of the transmitted signal through the rotation of the QAM constellation and making these components fade independently by means of a cyclic delay of the $\mathrm{Q}$ component. The rotation of the 
constellation allows casting every constellation symbol over I and Q axis independently in such a way that the I component contains intrinsic information of the $Q$ component and vice-versa. The cyclic delay of the $\mathrm{Q}$ component makes the I and $\mathrm{Q}$ components fade independently using a simplified approach of the signal space diversity (SSD) (Al-Semari \& Fuja, 1997). The insertion of a simple time delay for one of the two components avoids the loss of I and Q information due to a same fading event.

Fig. 12 shows the aforementioned process. Symbols are first mapped onto the constellation (white points) and then rotated (black points) to correlate the I and Q components. The resulting symbols are fed to a FEC block, after which the imaginary part is delayed one cell.

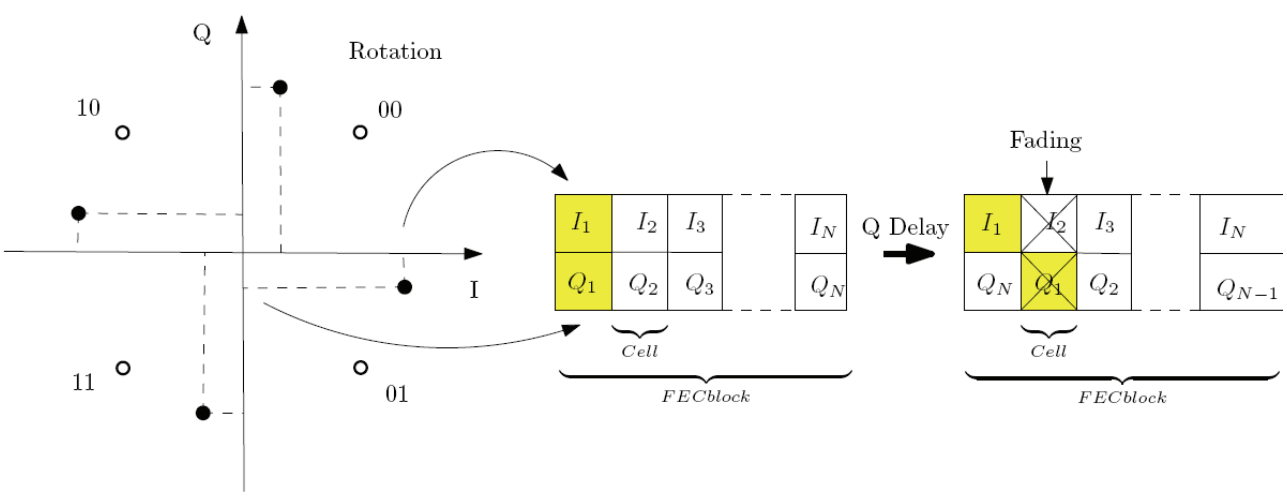

Fig. 12. Diagram of the rotated constellation and the $Q$ delay process.

\subsection{Analysis of RQD in the DVB-T2 BICM scheme over flat fading Rayleigh channel with erasures}

This section only considers the BICM module of DVB-T2 to study the effects of RQD on the performance of the overall system. In order to model a simple transmission with fading events, the approach to a flat Rayleigh channel with erasures (RME) based on (Nour \& Douillard, 2008) is assumed. The channel samples are considered uncorrelated due to all the interleaving modules of the DVB-T2 system. Hence, the equivalent received symbol $Y$ at the discrete instant $t$ is given by:

$$
Y(t)=H(t) E(t) X(t)+N(t)
$$

where $X(t)$ is the complex discrete transmit symbol, $H(t)$ is a Rayleigh distributed fading channel coefficient with $E[H(t)]=1, E(t)$ is a uniform random process which takes the value of zero with probability $P_{E}$ and $N(t)$ is the AWGN sample at time index $t$. The block diagram of Fig. 13 shows the simplified DVB-T2 BICM system over the RME channel

Fig. 14 presents the BER results of the DVB-T2 BICM system over a RME channel with $20 \%$ of erasures or lost subcarriers for two different code rates: $3 / 4$ and 2/3. For the first case, the LDPC cannot recover the lost information due to erasures and the performance of the system without RQD tends to an error floor above the QEF limit. However, the diversity added by RQD makes the LDPC correction possible providing a considerable improvement. On the other hand, when the error-correcting capacity of the code is greater, the improvement provided by RQD becomes smaller, as is shown in Fig. 14 for the case of a 


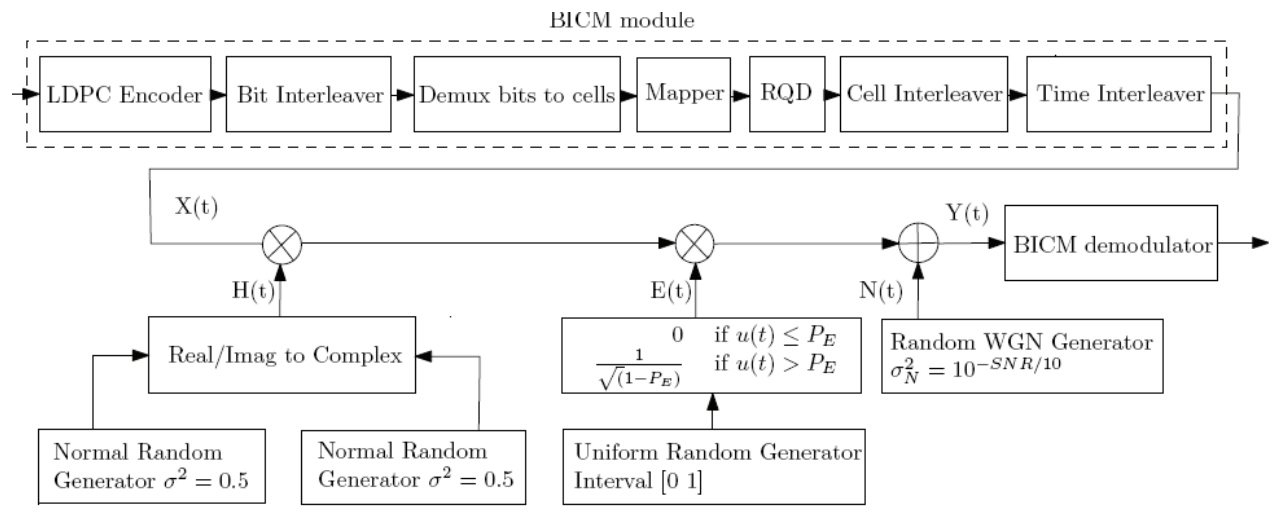

Fig. 13. Equivalent DVB-T2 BICM system over flat fading Rayleigh channel with erasures. code rate of $2 / 3$. Therefore, the gain of the system with RQD transmission results very significant over this kind of channel models for high coding rates and low modulation orders.

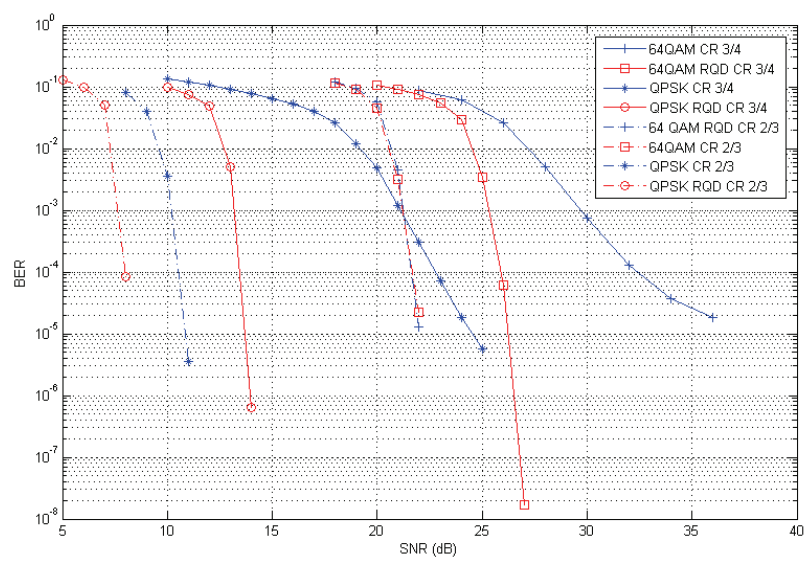

Fig. 14. Performance of RQD systems over flat Rayleigh channel with erasures of $20 \%$.

\subsection{Analysis of RQD in the DVB-T2 system over Rayleigh fading channels}

The complete DVB-T2 system model has now been used to analyze the behavior of the RQD technique. In this case, the channels are frequency-selective and hence every carrier of the OFDM symbol suffers a different fading event. Fig. 15 shows the results for Rayleigh Portable 1 (ETSI, 1997) and TU6 (COST207, 1989) channels. It can be seen that the gain of RQD decreases significantly in comparison to the results displayed in the previous section. This gain depends on the order of modulation and the code rate, decreasing when the latter is robust enough to recover the signal without RQD and increasing with the modulation order.

Fig. 15a depicts the RQD gain as a function of the modulation order considering a fixed code rate of 3/4. QPSK mode obtains a gain of $0.7 \mathrm{~dB}$, which is reduced to $0.3 \mathrm{~dB}$ in 16QAM and 
negligible for 64QAM. When the code rate is low, the RQD method improves the reception thanks to the increased diversity level. Consequently, the RQD method can be taken into account for high code rates where the redundancy rate of the coding is similar or even less than the loss of information. This is shown in Fig. 15b where the system with 16QAM modulation and code rate $5 / 6$ offers a gain of $0.5 \mathrm{~dB}$, whereas the gain disappears for the same modulation with code rate $3 / 5$. A very interesting conclusion that can be drawn from the results depicted in Fig. 15 is that the RME channel is not a very realistic channel when modeling realistic hard transmission scenarios like a TU6 channel.
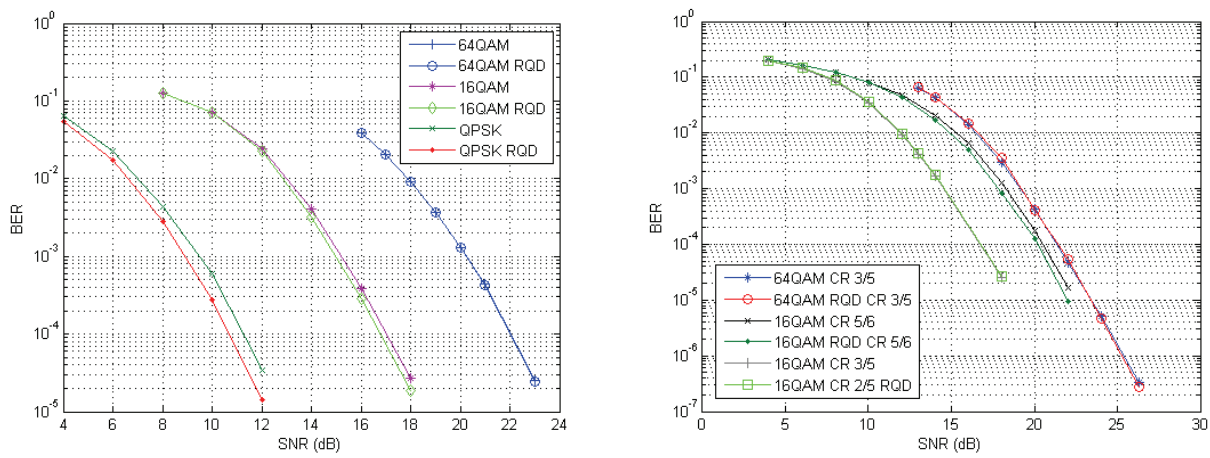

Fig. 15. Comparison of DVB-T2 systems in Rayleigh P1 channel with code rate 3/4 (a) and TU6 channel with code rates of $3 / 5$ and $5 / 6$ (b).

\section{Performance in single frequency networks}

DVB-T2 and its predecessor DVB-T are based on OFDM which is robust against intersymbol interference and fading caused by multipath propagation. These characteristics facilitate the deployment of SFN networks. Unlike multiple frequency networks (MFN), SFN involves several transmitters broadcasting synchronously the same program at the same frequency. The coverage area of every transmitter is called cell and the network can be composed of two or more cells which are deployed to cover wide areas with a unique frequency band.

The main advantage of this deployment strategy is the efficient use of the television spectrum, allowing a higher number of TV programs (Penttinen, 2008). On the other hand, the addition of two identical delayed signals is not always constructive and can lead to severe destructive interference in specific locations, making the signal unrecoverable. This section of the chapter evaluates the performance of DVB-T and DVB-T2 systems over SFN scenarios focusing on the benefits provided by the new standard.

\subsection{Self-interference analysis}

The simultaneous transmission from several sources can be considered as multipath propagation since the received echoes of the same signal form constructive or destructive interference which results in fading. This is known as self-interference and a correct SFN deployment is essential to reduce its degrading effects on the service area. The guard interval of the OFDM technique avoids ISI allowing operating in multipath fading 
environments. Nevertheless, although all the received signals of the SFN transmission are within the guard interval, their superposition may be destructive and the self-interference can degrade considerably the quality at reception. This effect can be avoided by means of directive antennas, but it is occasionally impossible due to environmental constraints. In that case, the performance of the receivers is reduced in locations with good signal reception level.

The SFN network gain is defined as the positive power contribution due to constructive superposition of all the received signals within the guard interval in comparison to the necessary power in order to cover the same area with a MFN network (Santella et al., 2004). However, as it has been explained previously, this contribution also generates destructive interference despite being inside the guard interval. The following simple constructive and destructive wave interference problem can justify this effect.

Using a phasor diagram as the one depicted in Fig. 16, the resulting waveform from the combination of two coherent sources can be expressed as the real part of the vector sum of the individual phasors with magnitude $A_{1}$ and $A_{2}$ and phases $\varphi_{1}$ and $\varphi_{2}$ respectively, which can be written as:

$$
\Psi_{T}(t)=A_{T} \cos \left(\omega t+\varphi_{T}\right)
$$

where the resultant waveform amplitude is:

$$
A_{T}=\sqrt{A_{1}^{2}+A_{2}^{2}+2 A_{1} A_{2} \cos \left(\varphi_{1}-\varphi_{2}\right)}
$$

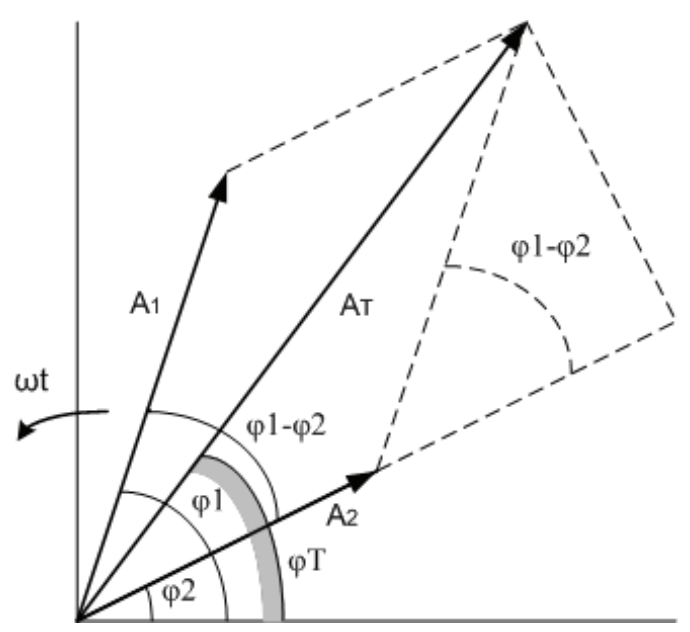

Fig. 16. Phasor diagram.

If $T_{0}$ is the resulting delay due to the phase differences, Equation (11) can be written as:

$$
A_{T}=\sqrt{A_{1}^{2}+A_{2}^{2}+2 A_{1} A_{2} \cos \left(2 \prod f T_{0}\right)}
$$

where $f$ is the carrier frequency. 
Equation (12) states that the resultant waveform amplitude will never be zero if received signal powers are different. However, if the power levels are equal, which involves that $A_{1}=A_{2}=A$, Equation (12) can be simplified after some operations as follows:

$$
A_{T}=2 A \cos \left(\Pi f T_{0}\right)
$$

In this case, the resultant waveform amplitude is zero periodically, meaning that several frequency subcarriers are lost. The number of nulls within the OFDM spectrum depends on the delay between transmitters since the distance between nulls is $\Delta f=1 / T_{0}$. Depending on the number of lost subcarriers and the code rate, this effect can avoid the QEF reception of the television signal.

\subsection{Performance of DVB-T/T2 systems in self-interference scenarios}

Simulation results are provided to analyze and compare the behavior of DVB-T and DVB-T2 systems in SFN networks due to the aforementioned self-interference scenario. The results, presented as BER over SNR curves, are achieved by means of Montecarlo simulations. The QEF limit after the convolutional decoder in DVB-T and after LDPC decoder in DVB-T2 is considered as an evaluation reference.

A DVB-T scenario is first considered where the receivers are at line of sight of two transmitters, being one of them delayed with different power levels. The synchronization to one transmitter is perfect, the direct component is only considered and an AWGN channel is assumed for simplicity. The Spanish DVB-T broadcasting options have been simulated as defined in the following table:

\begin{tabular}{|c|c|c|c|}
\hline FFT Size & Mode & Code Rate & GI \\
\hline 8K (8192 Carriers) & 64QAM & $2 / 3$ & $1 / 4$ \\
\hline
\end{tabular}

Table 2. Options of DVB-T simulations.

Fig. 17a and Fig. 17b show the behavior of the DVB-T system with echoes delayed for a half and a complete guard interval, respectively. Both situations provide an error floor in the performance for the $0 \mathrm{~dB}$ (same power) echo case. However, this keeps on appearing for -1 $\mathrm{dB}$ and $-2 \mathrm{~dB}$ in Fig. 17b. An echo delayed the guard interval length generates the most severe fading without introducing ISI and the number of cancelled carriers is twice as much as in the case of Fig. 17a. Furthermore, it can be seen that $0 \mathrm{~dB}$ and $-1 \mathrm{~dB}$ echoes generate error floors above the QEF limit.

The behavior of DVB-T2 in SFN networks has been evaluated with similar parameters as for DVB-T, as defined in the Table 3.

\begin{tabular}{|c|c|c|c|c|c|}
\hline FFT Size & Mode & $\begin{array}{c}\text { LDPC Block } \\
\text { Length }\end{array}$ & $\begin{array}{c}\text { LDPC Code } \\
\text { Rate }\end{array}$ & GI & $\begin{array}{c}\text { Pilot } \\
\text { Pattern }\end{array}$ \\
\hline 2K (2048 Carriers) & 64QAM & 16200 & $3 / 5$ & $1 / 4$ & PP1 \\
\hline
\end{tabular}

Table 3. Options of DVB-T2 simulations. 

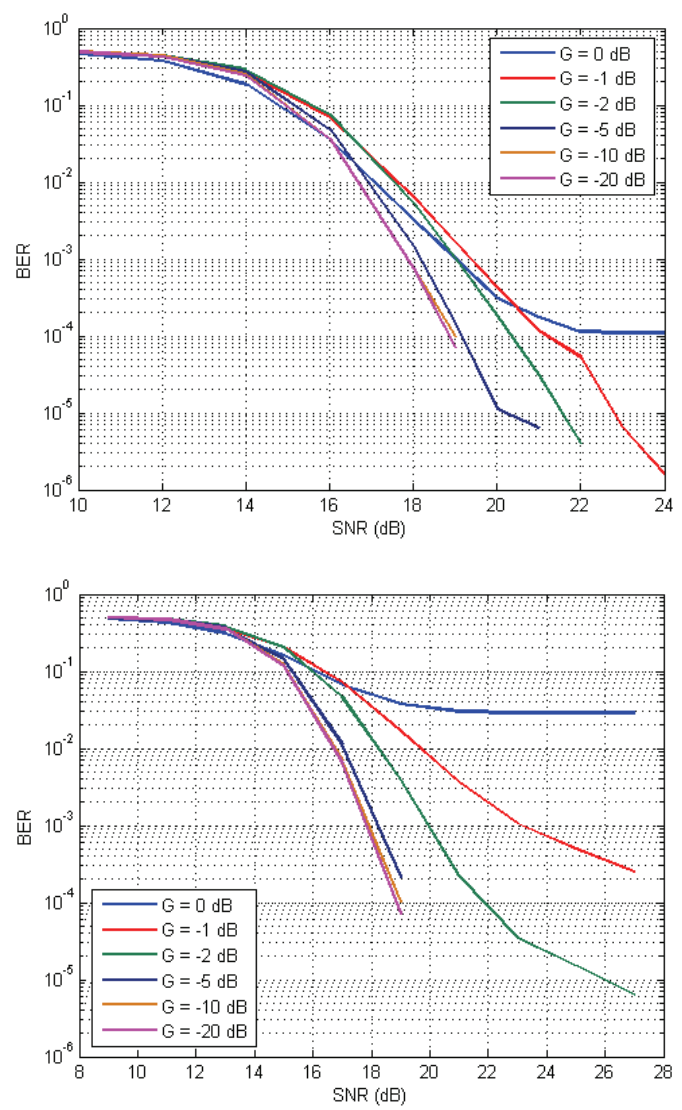

Fig. 17. BER performance of DVB-T system with an echo delayed GI/2 (a) and GI (b).

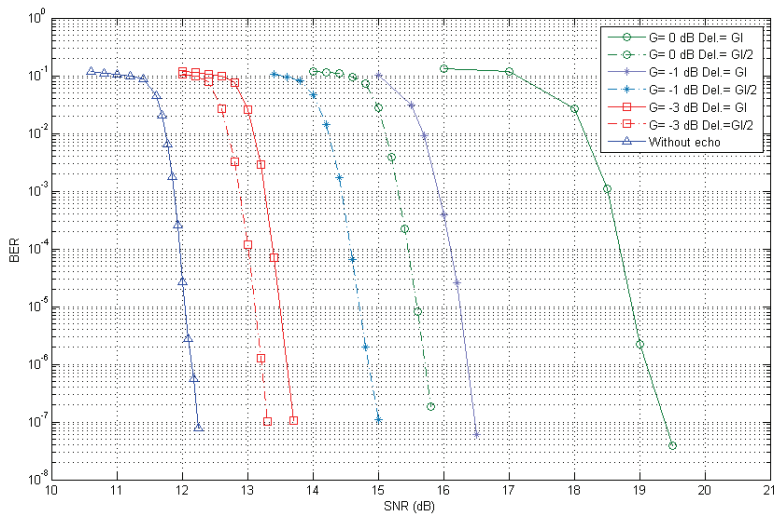

Fig. 18. DVB-T2 performance for echoes delayed GI/2. 
The FFT size is shorter in the case of DVB-T2 in order to reduce the simulation time, which does not affect the performance if delays are proportional. The optional RQD technique has been included since it adds diversity to the system.

Fig. 18 shows the simulation results with echoes of variable power delayed GI/2 and GI. One can see that the performance gain of the DVB-T2 system is around $6.5 \mathrm{~dB}$ greater than that of DVB-T and that it is more robust against self-interference. When the system is affected by an echo delayed GI, DVB-T2 achieves good reception where DVB-T does not reach QEF. As can be seen, all the simulated scenarios, which are as severe as in the previous DVB-T simulations, achieve the QEF limit of $10^{-7}$.

\section{Summary and conclusions}

This chapter has described the most interesting aspects of the new DVB-T2 digital video broadcasting standard. New research approaches and results have been shown regarding signal processing improvements over DVB-T2. A novel iterative demapping and decoding strategy has been shown which can be used with the LDPC codes provided by the new standard. Furthermore, results showing the effects of the number of demapping and LDPC iterations on the overall performance of the receiver have been provided. It has been proved that iterative demapping and detection, both regular and irregular, can improve considerably the performance of a DVB-T2 receiver, being specially suitable for problematic or low-level locations.

Multiantenna schemes and channel estimation algorithms have also been analyzed for the new standard, showing the importance of this issues on its behavior. The improvent achieved by the Alamouti MISO transmission scheme has been evaluated in conjunction with an MRC diversity scheme. This rather simple diversity approaches have shown a high perforance improvement in DVB-T2 scenarios.

Two special features of DVB-T2 have been specialy considered: the effects of the newly introduced rotated constellation transmission scheme and the performance of DVB-T2 in SFN scenarios. Regarding the rotated constellation approach its effects have been proven to be determinant in RME channels, whereas they introduce slight improvements in realistic frequency-selective channel like TU6. Considering the performance of the standard in a SFN interference scenario, DVB-T2 has shown to be a much more robust standard, allowing perfect reception of the signals in interference regions where DVB-T did not work.

\section{Acknowledgements}

The authors would like to thank the Spanish Government for the funding received through the research projects FURIA and FURIA2, as well as to all the partners of the FURIA consortium.

\section{References}

Alamouti, S. (1998). A simple transmit diversity technique for wireless communications. IEEE Journal on Selected Areas in Communications, Vol. 16, No. 8, Oct. 1998, pp 14511458, ISSN: 0733-8716. 
Al-Semari, S. \& Fuja, T. (1997). I-Q TCM: reliable communication over the Rayleigh fading cannel closet o the cutoff rate. IEEE Transactions on Information Theory, Vol. 43, No. 1, Jan. 1997, pp. 250-262.

Caire, G.; Taricco, G. \& Biglieri, E. (1998). Bit-interleaved coded modulation. IEEE Transactions on Information Theory, vol. 44, no. 3, May 1998, pp. 927-946.

COST207 (1989). Digital land mobile radio communications (Final report). Technical report, Commision of the European Communities, Directorate General Telecommunications, Information Industries and Innovation, 1989.

DVB (2008). Framing structure, channel coding and modulation for a second generation digital terrestrial television broadcasting system (DVB-T2). DVB Document A122, Jun. 2008.

DVB (2009). Implementation guidelines for a second generation digital terrestrial television broadcasting system (DVB-T2). DVB Document A133, Feb. 2009.

ETSI (1997). Digital video broadcasting (DVB); framing structure, channel coding and modulation for digital terrestrial television (DVB-T). ETS EN 300 744. Mar. 1997

Foschini, G. J. \& Gans, M. J. (1998). On limits of wireless communications in a fading environment when using multiple antennas. Wireless Personal Communications, Vol. 6, No. 3, Mar. 1998, pp. 311-335, ISSN: 0929-6212

Hoeher, P ; Kaiser, S; Robertson, P. Two-dimensional pilot-symbol-aided channel estimation by wiener filtering. IEEE International Conference on Acoustics, Speech, and Signal Processing, pp. 1845-1848, ISBN: 0-8186-7919-0, Munich, Germany, Apr. 1997.

Jakes, W. C. (1974). Microwave Mobile Communications. Wiley-IEEE Press. ISBN 0780310691. New York

Levy, Y. (2004). DVB-T - A fresh look at single and diversity receivers for mobile and portable reception. EBU Technical Review, No. 298, Apr. 2004.

Li, X.; Chindapol, A. \& Ritcey, J.A. (1998). Bit-interleaved coded modulation with iterative decoding using soft feedback. IEEE Electronic Letters, vol. 34, no. 10, May 1998, pp. 942-943.

Li, X.; Chindapol, A. \& Ritcey, J.A. (2002). Bit-interleaved coded modulation with iterative decoding and 8PSK signalling. IEEE Transactions on Communications, vol. 50, no. 8, Aug. 2002, pp. 1250-1257.

Necker, M ; Stüber, G. Totally blind channel estimation for OFDM on fast varying mobile radio channels. IEEE Transactions on Wireless Communications, vol. 3, no. 5, Sept. 2004, pp. 1514-1525. ISSN: 1536-1276.

Nour, C. A. \& Douillard, C. (2008). Rotated QAM constellations to improve BICM performance for DVB-T2. IEEE 10th International Symposium on Spread Spectrum Techniques and Applications, pp. 354-359, ISBN: 978-1-4244-2203-6, Bologna, Italy, Aug. 2008.

Patzold, M. (2002). Mobile Fading Channel. John Wiley \& Sons. ISBN 0471495492. Chichester, England.

Penttinen, J. T. J. (2008). The SFN gain in non-interfered and interfered DVB-H networks. The Fourth International Conference on Wireless and Mobile Communications, pp.294299, ISBN: 978-0-7695-3274-5, Athens, Greece, Jul. 2008. 
Proakis, J. G. (1995). Digital Communications. McGraw-Hill. ISBN 0072321113. New York, USA.

Santella, G.; De Martino, R. \& Ricchiuti, M. (2004). Single frequency network (SFN) planning for digital terrestrial television and radio broadcast services: the Italian frequency plan for T-DAB. IEEE 59th Vehicular Technology Conference, Vol. 4, May 2004, pp. 2307-2311, ISSN: 1550-2252.

Telatar, E. (1999). Capacity of multi-antenna Gaussian channels. European Transactions on Communications, Vol. 10, No. 6, Nov./Dec. 1999, pp. 585-595.

Zehavi, E. (1992). 8-PSK trellis codes for Rayleigh channel. IEEE Transactions on Communications, vol. 40, no. 5, May 1992, pp. 873-874. 


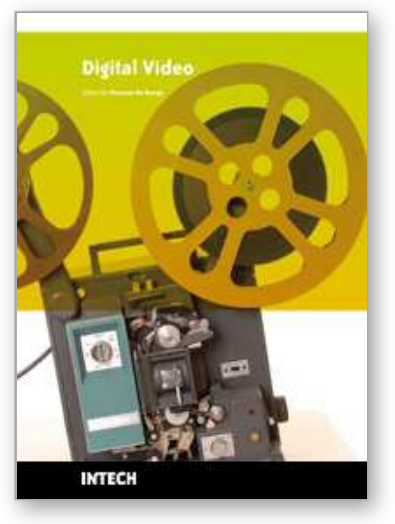

\author{
Digital Video \\ Edited by Floriano De Rango
}

ISBN 978-953-7619-70-1

Hard cover, 500 pages

Publisher InTech

Published online 01, February, 2010

Published in print edition February, 2010

This book tries to address different aspects and issues related to video and multimedia distribution over the heterogeneous environment considering broadband satellite networks and general wireless systems where wireless communications and conditions can pose serious problems to the efficient and reliable delivery of content. Specific chapters of the book relate to different research topics covering the architectural aspects of the most famous DVB standard (DVB-T, DVB-S/S2, DVB-H etc.), the protocol aspects and the transmission techniques making use of MIMO, hierarchical modulation and lossy compression. In addition, research issues related to the application layer and to the content semantic, organization and research on the web have also been addressed in order to give a complete view of the problems. The network technologies used in the book are mainly broadband wireless and satellite networks. The book can be read by intermediate students, researchers, engineers or people with some knowledge or specialization in network topics.

\title{
How to reference
}

In order to correctly reference this scholarly work, feel free to copy and paste the following:

Mikel Mendicute, Iker Sobrón, Lorena Martínez and Pello Ochandiano (2010). DVB-T2: New Signal Processing Algorithms for a Challenging Digital Video Broadcasting Standard, Digital Video, Floriano De Rango (Ed.), ISBN: 978-953-7619-70-1, InTech, Available from: http://www.intechopen.com/books/digital-video/dvb-t2-newsignal-processing-algorithms-for-a-challenging-digital-video-broadcasting-standard

\section{INTECH}

open science | open minds

\section{InTech Europe}

University Campus STeP Ri

Slavka Krautzeka 83/A

51000 Rijeka, Croatia

Phone: +385 (51) 770447

Fax: +385 (51) 686166

www.intechopen.com

\section{InTech China}

Unit 405, Office Block, Hotel Equatorial Shanghai

No.65, Yan An Road (West), Shanghai, 200040, China

中国上海市延安西路65号上海国际贵都大饭店办公楼 405 单元

Phone: +86-21-62489820

Fax: $+86-21-62489821$ 
(C) 2010 The Author(s). Licensee IntechOpen. This chapter is distributed under the terms of the Creative Commons Attribution-NonCommercialShareAlike-3.0 License, which permits use, distribution and reproduction for non-commercial purposes, provided the original is properly cited and derivative works building on this content are distributed under the same license. 